\title{
Encuesta Salud, Bienestar y Envejecimiento (SABE): metodología de la encuesta y perfil de la población estudiada
}

\author{
Cecilia Albala, ${ }^{1}$ Maria Lúcia Lebrão, ${ }^{2}$ Esther María León Díaz, ${ }^{3}$ \\ Roberto Ham-Chande, ${ }^{4}$ Anselm J. Hennis, ${ }^{5}$ Alberto Palloni, ${ }^{6}$ \\ Martha Peláez y Omar Pratts ${ }^{8}$
}

Forma de citar

Albala C, Lebrão ML, León Díaz EM, Ham-Chande R, Hennis AJ, Palloni A, et al. Encuesta Salud, Bienestar y Envejecimiento (SABE): metodología de la encuesta y perfil de la población estudiada. Rev Panam Salud Publica. 2005;17(5/6):307-22.

RESUMEN El presente documento reseña la metodología de la encuesta SABE y los desafíos que impone a la sociedad en general y a los servicios de salud en particular el rápido envejecimiento de la población en América Latina y el Caribe. La Región esta envejeciendo a un ritmo que no se ha observado en el mundo desarrollado, y la evaluación de problemas de salud y discapacidad indica que los adultos mayores están envejeciendo con más limitaciones funcionales y peor salud que sus semejantes en países desarrollados. Además, las redes familiares están cambiando rápidamente y tienen menos capacidad de suplir la falta de protección social institucional. El estudio multicéntrico $S A B E$ se creó con el objetivo de evaluar el estado de salud de las personas adultas mayores de siete ciudades de América Latina y el Caribe: Buenos Aires, Argentina; Bridgetown, Barbados; La Habana, Cuba; Montevideo, Uruguay; Santiago, Chile; México, D.F., México y São Paulo, Brasil. La encuesta SABE establece el punto de partida para la investigación sistemática del envejecimiento en zonas urbanas de la Región de América Latina y el Caribe. Se recomienda que estudios de estas características y con este ánimo comparativo se extiendan a otros países, zonas y regiones, para enriquecer el conocimiento sobre las personas adultas mayores.

Palabras clave Salud, calidad de vida, envejecimiento, población, América Latina, Región del Caribe.

El objetivo primario del estudio multicéntrico SABE fue evaluar el estado de salud de las personas adultas mayores de algunas ciudades de la Región, para

1 Instituto de Nutrición y Tecnologías de los Alimentos, Universidad de Chile. Santiago, Chile.

2 Faculdade de Saúde Pública, Universidade de São Paulo. São Paulo, Brasil.

3 Centro de Estudios de Población y Desarrollo (CEPDE). La Habana, Cuba.

4 El Colegio de la Frontera Norte. Tijuana, México.

5 Chronic Disease Research Centre, Tropical Medicine Research Institute, University of the West Indies, Bridgetown, Barbados.
6 Center for Demography and Ecology, University of Wisconsin at Madison, Wisconsin.

7 Organización Panamericana de la Salud. Washington, D.C., Estados Unidos de América. Toda correspondencia deberá dirigirse a: Martha Peláez, 525 23rd Street NW, Washington, DC 20037. EUA. Correo electrónico: pelaezma@paho.org

8 Centro de Estudios de Población, Universidad de Uruguay, Montevideo, Uruguay. proyectar las necesidades de salud que es probable que resulten del rápido crecimiento de la población adulta mayor. El objetivo secundario fue promover un mayor diálogo entre la investigación en salud pública y el estudio del envejecimiento, a fin de fortalecer un trabajo interdisciplinario en el que colaborarían epidemiólogos, demógrafos, sociólogos y geriatras. Las ciudades incluidas en el estudio SABE representan las diferentes 
etapas del envejecimiento en la Región. Cuatro de las ciudades incluidas (Buenos Aires en Argentina, Bridgetown en Barbados, La Habana en Cuba y Montevideo en Uruguay) se encuentran en una etapa avanzada del proceso de envejecimiento, mientras que las tres restantes (Santiago en Chile, México, D.F. en México y São Paulo en Brasil) están en una etapa un poco menos avanzada. Esta combinación de ciudades representa dos diferentes ritmos del envejecimiento de la Región. Dada la limitación de los recursos, no se incluyeron ciudades que están en una transición demográfica inicial, como las capitales de Bolivia, Perú, Guatemala u Honduras, lo cual queda como parte de un programa aún inconcluso de investigación en la Región. La encuesta SABE pone de manifiesto el compromiso institucional de la Organización Panamericana de la Salud con el tema del envejecimiento, y su éxito se debe a la colaboración y contribución de un gran número de personas e instituciones. ${ }^{9}$

\section{MARCO TEÓRICO PARA LA INVESTIGACIÓN SOBRE SALUD, BIENESTAR Y ENVEJECIMIENTO EN AMÉRICA LATINA Y EL CARIBE}

Las importantes repercusiones que ejerce el proceso de envejecimiento en el funcionamiento de las sociedades y en el bienestar afectan no solo a los adultos mayores, sino también a las personas más jóvenes (1-3). La influencia que los aspectos económicos, sociales y familiares tienen en el bienestar de las personas adultas mayo-

\footnotetext{
9 La Organización Panamericana de la Salud inició y coordinó el estudio en el nivel regional. El Centro de Demografía y Ecología de la Universidad de Wisconsin en Madison apoyó el desarrollo de la encuesta SABE. Las entrevistas se realizaron en las ciudades mencionadas, con el apoyo financiero de las siguientes instituciones: Organización Panamericana de la Salud (OPS), National Institute on Aging (NIA), Banco Interamericano de Desarrollo (BID), Banco de Desarrollo del Caribe, Fundação para o Amparo à Pesquisa do Estado de São Paulo (FAPES) en Brasil, Ministerio de Salud y BID en Santiago, Chile, Centro de Población del Instituto Nacional de Estadística y el Ministerio de Salud en La Habana, Cuba, Secretaría de Salud y Eli Lilly Inc. en México, y Ministerio de Salud y BID en Montevideo, Uruguay.
}

res varía según las peculiaridades demográficas, el desarrollo económico y la adecuación de los sistemas de salud y bienestar social para dar respuestas a los nuevos retos que impone el envejecimiento de la población. Uno de los factores de mayor importancia y trascendencia que sin duda afectará a los sistemas de salud de la Región durante el próximo decenio serán las condiciones de salud de los adultos mayores. El deterioro gradual de las condiciones de salud física y mental que acompaña al proceso de envejecimiento (4-6), la reducción resultante de los años esperados de vida activa y sana $(7,8)$, la reducción o el cese completo de la participación en el mercado laboral (9) y la mayor dependencia de las transferencias de ingresos de diversas fuentes públicas y privadas (10) determinan, en conjunto, que el crecimiento de la población de adultos mayores origine una mayor demanda de asistencia y servicios de salud. Dado que los trastornos de salud característicos de la edad avanzada son más crónicos que agudos y más progresivos que regresivos (11), esta demanda acarrea también un aumento excesivo de los costos de la asistencia sanitaria. Ejemplos de ello son los casos de Estados Unidos, Inglaterra y la mayor parte de los países de Europa Occidental, donde estos costos alcanzan magnitudes muy grandes $(12,13)$. Por otra parte, el caso de los países de Europa Oriental ha demostrado que la incapacidad de afrontar estos problemas se traduce en un rápido deterioro del estado de salud de los adultos mayores y en una pérdida sorprendente de la esperanza de vida $(14,15)$.

La tasa de crecimiento de la población mayor de 60 años es función de tres características: a) cambios en las tasas de natalidad pasadas, b) cambios en la mortalidad de los grupos de edad de 0 a 60 años en el pasado y, por último, c) cambios en la mortalidad del grupo de los mayores de 60 años. Está plenamente demostrado que las tasas de crecimiento actuales y futuras de la población adulta mayor se deben al descenso pasado de la mortalidad en los grupos de edad de 0 a 60 años. A fines del año 2000, en Argentina,
Barbados, Cuba y Uruguay la proporción de la población de 60 años y más se situaba entre 13 y 17\%; en Estados Unidos y Canadá la cifra estaba entre 16 y 17\%. En la mayor parte de los demás países de América del Sur, Centroamérica y el Caribe, la población de 60 años o más alcanzará o superará la cifra de $13 \%$ en los próximos 10 años. Las proyecciones actuales de la Organización de las Naciones Unidas indican que para 2025 América Latina y el Caribe habrán experimentado un envejecimiento sustancial de su estructura por edades; es decir, su población de 60 años y más habrá superado un promedio de 8 a 14\% (16).

Estas proyecciones demográficas dan motivo a la siguiente pregunta: ¿De qué manera el pasado incide en la salud futura y el estado de discapacidad de las personas adultas mayores? Una parte considerable de los aumentos futuros en la población de 60 años y más se atribuye a cambios experimentados en la mortalidad durante el período de 1930-1990. Estos cambios en la mortalidad se relacionan principalmente con la disminución de la frecuencia de las enfermedades infecciosas durante los primeros 10 años de la vida. Por tanto, el rápido envejecimiento que se registra en la Región puede explicarse, en parte, por las mejoras en salud pública que experimentaron muchos de los países latinoamericanos y que desencadenaron un pronunciado descenso de la mortalidad. En primer lugar, las mejoras en la supervivencia - ante todo en los primeros años de vida- que no se acompañaron de mejoras socioeconómicas probablemente darán lugar a cambios importantes en el promedio y la varianza de la distribución de la fragilidad de la población adulta mayor. Una línea nueva de investigación ha aportado pruebas de que la exposición a enfermedades o circunstancias nocivas en los primeros años de vida puede tener efectos fisiológicos duraderos que podrían influir durante la vida adulta de la persona (17). Tales consideraciones indican que las cohortes de personas adultas mayores que alcanzarán la edad de 60 años después del año 2000 son aquellas que experimen- 
taron, en el período posterior a la Segunda Guerra Mundial, los beneficios de una salud pública con mejores instrumentos y recursos, pero sin ganancias sustantivas en los ingresos económicos y la calidad de vida.

En resumen, la Región está envejeciendo con una rapidez que no tiene paralelo en el mundo desarrollado, de tal manera que la composición por estado de salud y discapacidad tiende a convertirse, tarde o temprano, en un problema grave y muy desfavorable. Además, las redes familiares están experimentando una rápida evolución y tienen menos capacidad de suplir la falta de protección social institucional (18). Una diferencia importante entre los países de la Región y las naciones más desarrolladas es la relación que existe entre la rapidez y la inercia del envejecimiento. Por un lado, los contextos económicos y sociales de las sociedades donde el proceso se lleva a cabo son determinantes. Por el otro, el proceso de envejecimiento en los países desarrollados se produjo después de alcanzarse altos niveles de vida, que redujeron las desigualdades económicas y sociales y motivaron la aplicación de estrategias institucionales para compensar el efecto residual de las desigualdades aún existentes, en particular en lo referente al acceso a los servicios de salud.

\section{ASPECTOS METODOLÓGICOS DE LA ENCUESTA}

El universo de estudio fue la población mayor de 60 años residente en hogares privados de residencia permanente en cada una de las ciudades seleccionadas. En tres de ellas se tuvo en cuenta la población periférica urbana, por considerarla parte integral de la ciudad, según el propio esquema de las oficinas de estadística nacionales.

En la mayor parte de las ciudades participantes del estudio SABE se efectúan encuestas nacionales periódicas de hogares y empleos, las cuales ofrecen marcos muestrales actualizados, por lo menos más recientes que los últimos censos disponibles (cuadro 1). La excepción fue Santiago, Chile, donde el marco muestral fue el censo de 1992. En Bridgetown, Barbados, el marco muestral fue el Registro Nacional Electoral, que se actualiza periódicamente.

Las muestras se elaboraron por el método clásico de muestreo de etapas múltiples por conglomerados, con estratificación de las unidades en los niveles más altos de agregación. En todos los países, excepto Barbados y Brasil, la muestra se seleccionó en tres etapas. Solo en Barbados y Brasil se aplicaron dos etapas de selección. El esquema que se siguió en cada caso fue bastante similar. La unidad primaria de muestreo (UPM) fue un conglomerado de hogares independientes, dentro de una zona geográfica determinada. Las UPM se agruparon en estratos geográficos o socioeconómicos. La distribución de la muestra por estrato geográfico o socioeconómico se determinó mediante una asignación proporcional al tamaño de la población adulta mayor. Las UPM se dividieron a su vez en unidades secundarias de muestreo (USM), cada una formada por un número más pequeño de hogares independientes. Estas USM se integraron a su vez por unidades terciarias de muestreo (UTM), constituidas por los hogares seleccionados en que se entrevistó a todas las personas de 60 años o más residentes en ellos, o por individuos, en aquellos países en que se seleccionó solo a una persona en cada hogar. De esta manera, el hogar o la persona objetivo (entrevistada) constituyó el último estrato de agregación en la muestra.

Las etapas del diseño permitieron al investigador calcular las probabilidades de selección de cada individuo objetivo. Esto, junto con el conocimiento ex post de las tasas de falta de respuesta, aportó la información suficiente para calcular los factores de expansión, a fin de realizar inferencias respecto de la población de referencia a partir de las muestras seleccionadas.

Existen diferencias importantes en el diseño muestral entre las ciudades. La primera es la estratificación de los conglomerados. En algunas ciudades la estratificación se efectuó solo en términos geográficos, mientras que en otras los estratos se definieron en términos geo- gráficos y por indicadores socioeconómicos agregados. En el caso de Bridgetown, los hogares se estratificaron de acuerdo con la proporción de personas mayores de 60 o de 80 años.

El segundo aspecto diferente es la naturaleza del sobremuestreo realizado. En cuatro ciudades las muestras incluyeron un sobremuestreo de la población mayor de 80 años. En La Habana y Montevideo se seleccionó siempre a una persona mayor de 80 años. En Santiago, Chile, la persona objetivo se seleccionó aleatoriamente entre los miembros del hogar, pero si una persona mayor de 80 años estaba presente y no resultaba elegida por el proceso de selección aleatoria, entonces también se entrevistaba a esta persona. En São Paulo se diseñó una muestra adicional para entrevistar a personas mayores de 75 años.

La tercera diferencia se refiere a la última etapa de selección o la unidad de muestreo. En cinco ciudades (Buenos Aires, Bridgetown, Santiago, La Habana y Montevideo) se seleccionó para la entrevista solamente a una persona en cada hogar. En dos de estas ciudades, La Habana y Bridgetown, se entrevistó también a los cónyuges sobrevivientes y se les incluyó en una base adicional para hacer estudios en parejas. $^{10}$

En dos ciudades, México, D.F. y São Paulo, se entrevistó a todas las personas residentes en el hogar que cumplían los requisitos, incluidos sus cónyuges, si estaban presentes. Por último, como se mencionó, en Santiago se seleccionó al azar para la entrevista solamente a una persona entre todas las que cumplían los requisitos y, además, se realizó una segunda entrevista con las personas mayores de 80 años, si alguna estaba presente, aun cuando no se hubiera seleccionado mediante el proceso aleatorio preestablecido.

Para estimar los errores muestrales y los efectos de diseño se emplearon procedimientos convencionales, basados en pesos muestrales, estratificación y

\footnotetext{
10 En Cuba, el cuestionario del cónyuge fue una versión reducida del cuestionario del individuo objetivo. Además, los cónyuges no proporcionaron las medidas antropométricas.
} 
CUADRO 1. Diseño de la muestra, por ciudades

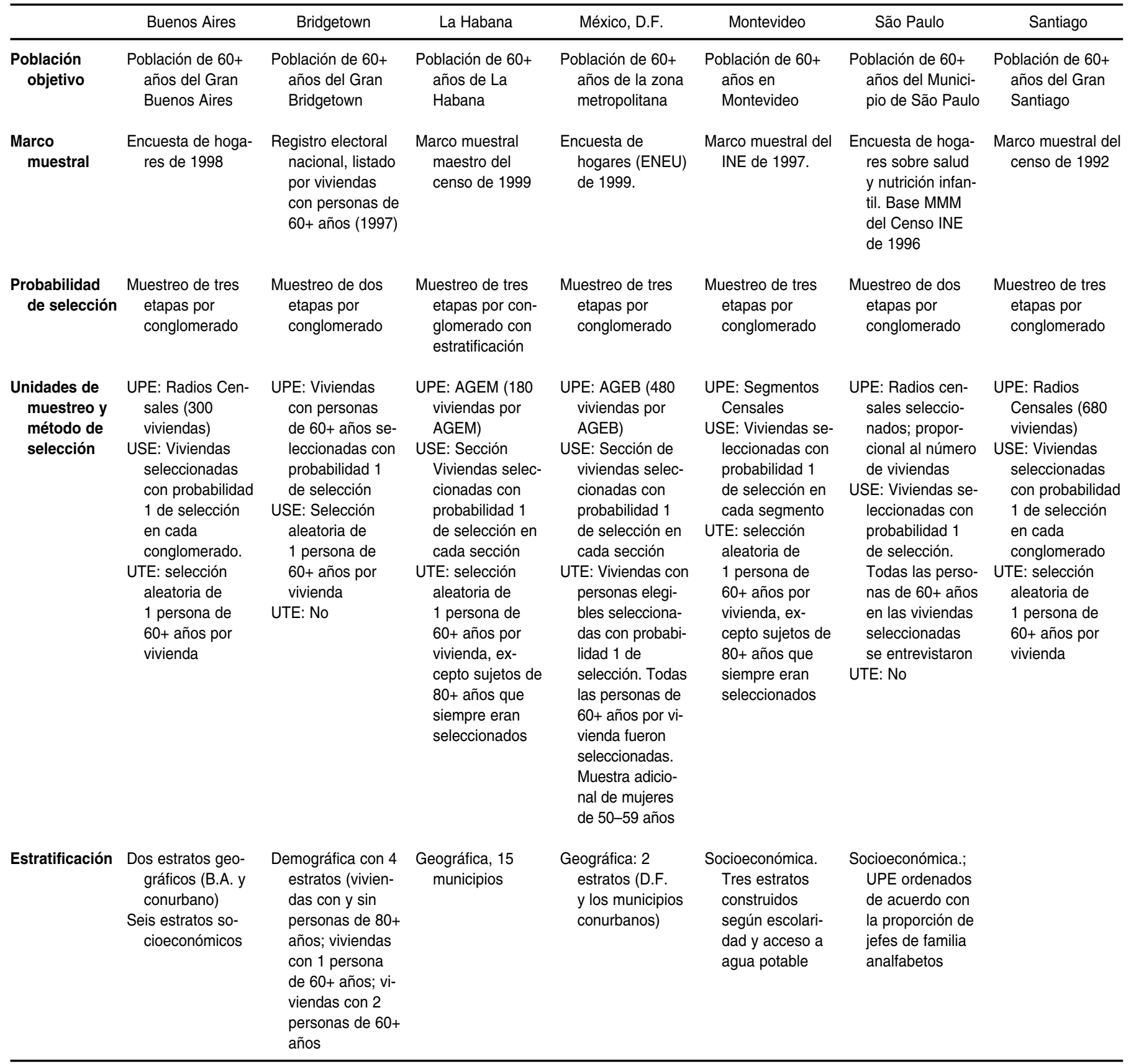

Fuente: Organización Panamericana del la Salud. Encuesta SABE, 2001 e informes preliminares.

Nota: UPE = unidades de primera etapa; USE = unidades de segunda etapa; UTE = unidades de tercera etapa.

tipo de UPM, así como sus conglomerados. A continuación se presentan las estimaciones de los errores muestrales y efectos de diseño de algunas variables, desagregadas por sexo. Los resultados se resumen en los cuadros 2 a 9.

Dadas las peculiaridades de cada ciudad, fue necesario modificar las expresiones convencionales para el cálculo de los errores muestrales y efectos de diseño. En las ciudades en que se hizo un muestreo aleatorio simple, la UPM fue pareada con estratificación pero no con los conglomerados de las UPM (Santiago, Chile, y Bridgetown, Barbados). En el caso de México, D.F., varios estratos estuvieron representados por una sola UPM, de modo que no fue posible calcular la varianza dentro de los estratos. Se decidió agrupar las correspondientes UPM en un solo estrato ficticio para poder calcular las varianzas y efectos de diseño. Con esto se obtuvo un límite superior 
para los efectos de diseño y los errores muestrales. ${ }^{11}$

El cuestionario se diseñó de tal manera que aportara información comparable entre los varios países. En particular, el objetivo fue incluir módulos y secciones basadas en la Health and Retirement Survey (Encuesta de Salud y Jubilación) $^{12}$ y la Encuesta AHEAD, ${ }^{13}$ además de un conjunto de encuestas realizadas por el Centro de Estudios de Población de la Universidad de Michigan ${ }^{14}$ y sus colaboradores locales en varios países de Asia. Al tiempo que se buscó fortalecer la comparabilidad, se procuró que los módulos incluidos fuesen congruentes con la realidad de cada ciudad que se investigaba. El instrumento final se consideró apropiado para la identificación de lo que es pertinente al proceso de envejecimiento en las grandes zonas urbanas de la Región y de las semejanzas que estas guardan con las de otros países.

El cuestionario se divide en varios módulos, a saber: características demográficas básicas, características socioeconómicas de la persona, pertenencia al hogar y características de la vivienda, autoevaluación de la salud y enfermedades crónicas, uso y acceso a los servicios de salud, medicamentos, cognición y depresión, evaluación nutricional, actividades de la vida diaria (AVD) y actividades instrumentales de la vida diaria (AIVD), antecedentes laborales e ingresos, propiedad y activos, transferencias familiares e institucionales.

El cuestionario fue solo uno de dos componentes del protocolo para reunir información. El segundo consistió en medidas antropométricas (peso, estatura, altura de rodilla, circunferencia del brazo, circunferencia de la cintura y la cadera, pliegue tricipital, circunferencia de la pantorrilla y ancho de la muñeca). También se aplicaron algunas pruebas sencillas para evaluar el estado funcional. Entre ellas se le pidió

\footnotetext{
11 La experimentación con soluciones alternativas ha demostrado que las estimaciones son insensibles a la presencia de estos estratos apartados con UPM únicos.

12 http://hrsonline.isr.umich.edu/

13 http://hrsonline.isr.umich.edu/

14 http://www.psc.isr.umich.edu/
}

CUADRO 2. Distribución porcentual de los adultos mayores por sexo, grupos de edad (en años) y ciudades de estudio, 2001

\begin{tabular}{|c|c|c|c|c|c|c|c|c|}
\hline \multirow[b]{2}{*}{ Ciudad } & \multicolumn{2}{|c|}{60 años y más } & \multicolumn{3}{|c|}{ Mujeres } & \multicolumn{3}{|c|}{ Varones } \\
\hline & Mujeres & Varones & $60-64$ & $65-74$ & $75+$ & $60-64$ & $65-74$ & $75+$ \\
\hline Buenos Aires & 62 & 38 & 21,52 & 49,94 & 28,55 & 22,82 & 51,22 & 25,96 \\
\hline Bridgetown & 60 & 40 & 20,79 & 42,51 & 36,69 & 15,62 & 46,99 & 37,40 \\
\hline São Paulo & 58 & 42 & 30,80 & 45,00 & 24,20 & 34,40 & 46,43 & 19,17 \\
\hline Santiago & 66 & 34 & 27,29 & 42,40 & 30,31 & 32,88 & 43,38 & 23,73 \\
\hline La Habana & 59 & 41 & 26,24 & 39,78 & 33,98 & 30,39 & 43,14 & 26,47 \\
\hline México, D.F. & 56 & 44 & 29,48 & 43,55 & 26,97 & 36,10 & 43,56 & 20,34 \\
\hline Montevideo & 64 & 36 & 21,91 & 47,06 & 31,03 & 20,00 & 51,48 & 28,52 \\
\hline
\end{tabular}

Fuente: Organización Panamericana de la Salud. Encuesta SABE, 2001 e informes preliminares.

CUADRO 3. Variables seleccionadas descriptivas de la población encuestada en Buenos Aires (Argentina), por sexo

\begin{tabular}{|c|c|c|c|c|c|c|}
\hline Variable & $\begin{array}{c}\text { Estimador } \\
\text { MUJER }\end{array}$ & $\begin{array}{c}\text { Error } \\
\text { estándar }\end{array}$ & IC95\% ${ }^{a}$ & $\begin{array}{l}\text { Estimador } \\
\text { HOMBRE }\end{array}$ & $\begin{array}{c}\text { Error } \\
\text { estándar }\end{array}$ & IC95\% \\
\hline \multicolumn{7}{|l|}{ Edad (años) } \\
\hline 60-64 & 0,215 & 0,017 & $0,181-0,249$ & 0,228 & 0,021 & $0,186-0,270$ \\
\hline $65-74$ & 0,499 & 0,021 & $0,458-0,541$ & 0,512 & 0,027 & $0,458-0,566$ \\
\hline 75 o más & 0,285 & 0,018 & $0,249-0,322$ & 0,260 & 0,025 & $0,211-0,308$ \\
\hline \multicolumn{7}{|l|}{ Escolaridad } \\
\hline Sin educación formal & 0,041 & 0,008 & $0,026-0,057$ & 0,019 & 0,007 & $0,004-0,033$ \\
\hline Primaria & 0,697 & 0,020 & $0,658-0,735$ & 0,611 & 0,027 & $0,557-0,664$ \\
\hline Secundaria o más & 0,262 & 0,019 & $0,225-0,299$ & 0,371 & 0,027 & $0,318-0,423$ \\
\hline \multicolumn{7}{|l|}{ Estado civil } \\
\hline Nunca casado & 0,062 & 0,011 & $0,041-0,084$ & 0,040 & 0,009 & $0,022-0,058$ \\
\hline En unión libre & 0,433 & 0,022 & $0,390-0,475$ & 0,744 & 0,021 & $0,702-0,786$ \\
\hline Divorciado, separado, viudo & 0,504 & 0,021 & $0,463-0,545$ & 0,213 & 0,020 & $0,174-0,251$ \\
\hline \multicolumn{7}{|l|}{ Fuerza de trabajo } \\
\hline Activo & 0,158 & 0,015 & $0,129-0,188$ & 0,374 & 0,026 & $0,322-0,426$ \\
\hline \multicolumn{7}{|l|}{ Estado de salud declarado } \\
\hline Muy bueno & 0,155 & 0,016 & $0,125-0,186$ & 0,252 & 0,024 & $0,205-0,300$ \\
\hline Bueno & 0,437 & 0,021 & $0,396-0,478$ & 0,444 & 0,027 & $0,390-0,497$ \\
\hline Regular & 0,315 & 0,019 & $0,277-0,353$ & 0,239 & 0,024 & $0,192-0,285$ \\
\hline Malo & 0,061 & 0,010 & $0,041-0,081$ & 0,038 & 0,010 & $0,017-0,058$ \\
\hline \multicolumn{7}{|l|}{ Salud mental } \\
\hline Depresión & 0,152 & 0,015 & $0,123-0,181$ & 0,112 & 0,018 & $0,077-0,146$ \\
\hline Deterioro cognoscitivo & 0,075 & 0,010 & $0,055-0,096$ & 0,060 & 0,014 & $0,033-0,087$ \\
\hline \multicolumn{7}{|l|}{ Problemas de salud crónicos } \\
\hline \multicolumn{7}{|l|}{ Bajo peso } \\
\hline \multicolumn{7}{|l|}{ Obesidad } \\
\hline Artritis & 0,627 & 0,020 & $0,587-0,667$ & 0,365 & 0,027 & $0,312-0,417$ \\
\hline Hipertensión & 0,510 & 0,021 & $0,469-0,551$ & 0,475 & 0,028 & $0,420-0,529$ \\
\hline Diabetes & 0,114 & 0,013 & $0,088-0,139$ & 0,140 & 0,019 & $0,102-0,177$ \\
\hline Cáncer & 0,060 & 0,010 & $0,040-0,080$ & 0,041 & 0,011 & $0,019-0,063$ \\
\hline Enfermedades pulmonares & 0,065 & 0,009 & $0,045-0,082$ & 0,103 & 0,017 & $0,070-0,135$ \\
\hline Enfermedades del corazón & 0,191 & 0,016 & $0,160-0,223$ & 0,210 & 0,023 & $0,165-0,254$ \\
\hline \multicolumn{7}{|l|}{ Discapacidad } \\
\hline AVDb (1 o más) & 0,202 & 0,016 & $0,170-0,234$ & 0,128 & 0,018 & $0,092-0,164$ \\
\hline AIVDc (1 o más) & 0,222 & 0,017 & $0,189-0,255$ & 0,096 & 0,016 & $0,065-0,127$ \\
\hline \multicolumn{7}{|l|}{ Arreglos domiciliarios } \\
\hline Solo & 0,231 & 0,015 & $0,201-0,261$ & 0,139 & 0,016 & $0,108-0,170$ \\
\hline Con pareja & 0,431 & 0,022 & $0,390-0,474$ & 0,740 & 0,022 & $0,698-0,782$ \\
\hline Con otras personas & 0,337 & 0,019 & $0,300-0,375$ & 0,121 & 0,016 & $0,090-0,152$ \\
\hline
\end{tabular}

Fuente: Organización Panamericana de la Salud. Encuesta SABE, 2001 e informes preliminares.

a IC95\%: intervalo de confianza de $95 \%$.

b Actividades de la vida diaria.

${ }^{c}$ Actividades instrumentales de la vida diaria. 
CUADRO 4. Variables seleccionadas descriptivas de la población encuestada en Bridgetown (Barbados), por sexo

\begin{tabular}{|c|c|c|c|c|c|c|}
\hline Variable & $\begin{array}{l}\text { Estimador } \\
\text { MUJER }\end{array}$ & $\begin{array}{c}\text { Error } \\
\text { estándar }\end{array}$ & $\mathrm{IC} 95 \% \mathrm{a}$ & $\begin{array}{l}\text { Estimador } \\
\text { HOMBRE }\end{array}$ & $\begin{array}{c}\text { Error } \\
\text { estándar }\end{array}$ & IC95\% \\
\hline \multicolumn{7}{|l|}{ Edad (años) } \\
\hline $60-64$ & 0,198 & 0,013 & $0,172-0,224$ & 0,206 & 0,018 & $0,170-0,242$ \\
\hline $65-74$ & 0,393 & 0,016 & $0,361-0,425$ & 0,445 & 0,021 & $0,404-0,485$ \\
\hline 75 o más & 0,409 & 0,016 & $0,377-0,441$ & 0,349 & 0,020 & $0,311-0,388$ \\
\hline \multicolumn{7}{|l|}{ Escolaridad } \\
\hline Sin educación formal & 0,008 & 0,003 & $0,002-0,013$ & 0,008 & 0,004 & $0,001-0,015$ \\
\hline Primaria & 0,774 & 0,014 & $0,747-0,801$ & 0,753 & 0,018 & $0,718-0,789$ \\
\hline Secundaria o más & 0,216 & 0,014 & $0,189-0,243$ & 0,230 & 0,018 & $0,195-0,265$ \\
\hline \multicolumn{7}{|l|}{ Estado civil } \\
\hline Nunca casado & 0,258 & 0,015 & $0,230-0,287$ & 0,127 & 0,014 & $0,100-0,155$ \\
\hline En unión libre & 0,231 & 0,014 & $0,204-0,259$ & 0,522 & 0,021 & $0,481-0,563$ \\
\hline Divorciado, separado, viudo & 0,498 & 0,017 & $0,465-0,530$ & 0,341 & 0,020 & $0,302-0,380$ \\
\hline \multicolumn{7}{|l|}{ Fuerza de trabajo } \\
\hline Activo & 0,118 & 0,011 & $0,097-0,139$ & 0,213 & 0,018 & $0,178-0,248$ \\
\hline \multicolumn{7}{|l|}{ Estado de salud declarado } \\
\hline Muy bueno & 0,121 & 0,011 & $0,100-0,143$ & 0,204 & 0,017 & $0,170-0,237$ \\
\hline Bueno & 0,335 & 0,016 & $0,304-0,366$ & 0,375 & 0,020 & $0,335-0,415$ \\
\hline Regular & 0,485 & 0,017 & $0,452-0,517$ & 0,371 & 0,020 & $0,331-0,410$ \\
\hline Malo & 0,055 & 0,008 & $0,040-0,070$ & 0,050 & 0,009 & $0,033-0,068$ \\
\hline \multicolumn{7}{|l|}{ Salud mental } \\
\hline Depresión & 0,044 & 0,007 & $0,031-0,058$ & 0,055 & 0,010 & $0,035-0,074$ \\
\hline Deterioro cognoscitivo & 0,075 & 0,009 & $0,058-0,092$ & 0,055 & 0,010 & $0,037-0,074$ \\
\hline \multicolumn{7}{|l|}{ Problemas de salud crónicos } \\
\hline Bajo peso & 0,041 & 0,007 & $0,028-0,053$ & 0,048 & 0,009 & $0,030-0,065$ \\
\hline Obesidad & 0,295 & 0,015 & $0,266-0,325$ & 0,112 & 0,013 & $0,086-0,138$ \\
\hline Artritis & 0,577 & 0,016 & $0,545-0,609$ & 0,316 & 0,019 & $0,278-0,354$ \\
\hline Hipertensión & 0,535 & 0,017 & $0,503-0,568$ & 0,378 & 0,020 & $0,338-0,418$ \\
\hline Diabetes & 0,236 & 0,014 & $0,208-0,263$ & 0,187 & 0,017 & $0,155-0,220$ \\
\hline Cáncer & 0,035 & 0,006 & $0,023-0,047$ & 0,022 & 0,006 & $0,010-0,033$ \\
\hline Enfermedades pulmonares & 0,046 & 0,007 & $0,033-0,060$ & 0,037 & 0,008 & $0,021-0,052$ \\
\hline Enfermedades del corazón & 0,114 & 0,011 & $0,094-0,135$ & 0,116 & 0,013 & $0,090-0,142$ \\
\hline \multicolumn{7}{|l|}{ Discapacidad } \\
\hline AVDb (1 o más) & 0,166 & 0,012 & $0,142-0,166$ & 0,109 & 0,013 & $0,084-0,135$ \\
\hline AIVDc (1 o más) & 0,243 & 0,014 & $0,215-0,243$ & 0,150 & 0,015 & $0,120-0,179$ \\
\hline \multicolumn{7}{|l|}{ Arreglos domiciliarios } \\
\hline Solo & 0,238 & 0,014 & $0,210-0,266$ & 0,268 & 0,019 & $0,231-0,304$ \\
\hline Con pareja & 0,252 & 0,014 & $0,224-0,281$ & 0,507 & 0,021 & $0,466-0,548$ \\
\hline Con otras personas & 0,510 & 0,017 & $0,477-0,542$ & 0,225 & 0,018 & $0,191-0,260$ \\
\hline
\end{tabular}

al adulto mayor que demostrara su capacidad para realizar las siguientes maniobras físicas:
a) sostenerse en un solo pie
b) agacharse y acuclillarse
c) levantarse y sentarse sin ayuda.

El Banco Interamericano de Desarrollo (BID) financió la inclusión de tres módulos especiales en el cuestionario central del estudio SABE. El módulo del BID tuvo preguntas sobre la economía doméstica y el consumo de bienes y servicios específicos en el hogar, las barreras ambientales, la demanda de recursos técnicos de apoyo, las actividades de recreación y el uso del tiempo libre. Estos módulos fueron incluidos al final de la encuesta en Santiago, Buenos Aires y Montevideo. El propósito de estos módulos fue evaluar la demanda de los mercados especiales para satisfacer las necesidades y deseos específicos de las personas de edad y su buena disposición para pagarlos.

Para lograr la mejor comparación posible entre encuestas se establecieron algunos principios generales, a los cuales se adhirieron todos los países. En primer lugar, los investigadores principales de cada ciudad fueron capacitados en cuanto al contenido, el proceso de la entrevista, su aplicación, la naturaleza de la muestra y la realización del trabajo de campo. En segundo lugar, en todos los casos la capacitación de los supervisores y de los encuestadores estuvo a cargo de los equipos principales de investigadores, con el apoyo del personal apropiado. En tercero, el trabajo de campo general se inició en octubre de 1999 en Santiago y terminó en mayo de 2001 
CUADRO 5. Variables seleccionadas descriptivas de la población encuestada en São Paulo (Brasil), por sexo

\begin{tabular}{|c|c|c|c|c|c|c|}
\hline Variable & $\begin{array}{l}\text { Estimador } \\
\text { MUJER }\end{array}$ & $\begin{array}{l}\text { Error } \\
\text { estándar }\end{array}$ & IC95\% ${ }^{a}$ & $\begin{array}{l}\text { Estimador } \\
\text { HOMBRE }\end{array}$ & $\begin{array}{l}\text { Error } \\
\text { estándar }\end{array}$ & IC95\% \\
\hline \multicolumn{7}{|l|}{ Edad (años) } \\
\hline $60-64$ & 0,308 & 0,018 & $0,272-0,344$ & 0,344 & 0,027 & $0,290-0,398$ \\
\hline $65-74$ & 0,450 & 0,020 & $0,410-0,490$ & 0,464 & 0,030 & $0,404-0,525$ \\
\hline 75 o más & 0,242 & 0,025 & $0,192-0,292$ & 0,192 & 0,029 & $0,133-0,251$ \\
\hline \multicolumn{7}{|l|}{ Escolaridad } \\
\hline Sin educación formal & 0,231 & 0,021 & $0,189-0,274$ & 0,180 & 0,019 & $0,142-0,219$ \\
\hline Primaria & 0,654 & 0,021 & $0,612-0,696$ & 0,634 & 0,028 & $0,578-0,691$ \\
\hline Secundaria o más & 0,109 & 0,020 & $0,069-0,148$ & 0,182 & 0,030 & $0,121-0,242$ \\
\hline \multicolumn{7}{|l|}{ Estado civil } \\
\hline Nunca casado & 0,052 & 0,008 & $0,036-0,069$ & 0,044 & 0,008 & $0,028-0,060$ \\
\hline En unión libre & 0,413 & 0,016 & $0,381-0,445$ & 0,791 & 0,018 & $0,756-0,826$ \\
\hline Divorciado, separado, viudo & 0,533 & 0,017 & $0,499-0,568$ & 0,165 & 0,016 & $0,132-0,198$ \\
\hline \multicolumn{7}{|l|}{ Fuerza de trabajo } \\
\hline \multirow{2}{*}{\multicolumn{7}{|c|}{ Estado de salud declarado }} \\
\hline & & & & & & \\
\hline Muy bueno & 0,106 & 0,010 & $0,086-0,127$ & 0,107 & 0,016 & $0,076-0,139$ \\
\hline Bueno & 0,336 & 0,019 & $0,298-0,375$ & 0,377 & 0,019 & $0,339-0,415$ \\
\hline Regular & 0,465 & 0,019 & $0,428-0,503$ & 0,444 & 0,019 & $0,406-0,482$ \\
\hline Malo & 0,089 & 0,008 & $0,072-0,106$ & 0,072 & 0,010 & $0,052-0,091$ \\
\hline \multicolumn{7}{|l|}{ Salud mental } \\
\hline Depresión & 0,233 & 0,013 & $0,207-0,258$ & 0,134 & 0,012 & $0,109-0,159$ \\
\hline Deterioro cognoscitivo & 0,131 & 0,010 & $0,111-0,150$ & 0,125 & 0,014 & $0,097-0,153$ \\
\hline \multicolumn{7}{|l|}{ Problemas de salud crónicos } \\
\hline Bajo peso & 0,017 & 0,004 & $0,009-0,026$ & 0,021 & 0,005 & $0,011-0,030$ \\
\hline Obesidad & 0,246 & 0,015 & $0,217-0,275$ & 0,091 & 0,010 & $0,071-0,111$ \\
\hline Artritis & 0,404 & 0,013 & $0,377-0,430$ & 0,208 & 0,015 & $0,177-0,239$ \\
\hline Hipertensión & 0,566 & 0,017 & $0,532-0,560$ & 0,495 & 0,021 & $0,452-0,537$ \\
\hline Diabetes & 0,187 & 0,012 & $0,163-0,212$ & 0,170 & 0,017 & $0,135-0,205$ \\
\hline Cáncer & 0,035 & 0,005 & $0,024-0,045$ & 0,031 & 0,006 & $0,018-0,044$ \\
\hline Enfermedades pulmonares & 0,107 & 0,010 & $0,087-0,127$ & 0,144 & 0,013 & $0,118-0,169$ \\
\hline Enfermedades del corazón & 0,189 & 0,013 & $0,164-0,214$ & 0,205 & 0,015 & $0,175-0,235$ \\
\hline \multicolumn{7}{|l|}{ Discapacidad } \\
\hline AVD ${ }^{b}$ (1 o más) & 0,223 & 0,014 & $0,194-0,252$ & 0,148 & 0,018 & $0,113-0,184$ \\
\hline AIVDc (1 o más) & 0,323 & 0,018 & $0,287-0,359$ & 0,183 & 0,016 & $0,152-0,215$ \\
\hline \multicolumn{7}{|l|}{ Arreglos domiciliarios } \\
\hline Solo & 0,170 & 0,012 & $0,145-0,195$ & 0,075 & 0,010 & $0,055-0,095$ \\
\hline Con pareja & 0,404 & 0,015 & $0,374-0,435$ & 0,782 & 0,017 & $0,748-0,817$ \\
\hline Con otras personas & 0,426 & 0,015 & $0,396-0,456$ & 0,142 & 0,014 & $0,113-0,172$ \\
\hline
\end{tabular}

en São Paulo. En su realización se utilizaron reglas similares, a fin de llevar al mínimo las pérdidas muestrales y hacer seguimiento de los casos difíciles de obtener, especialmente en ciudades en que se esperaban tasas de respuesta más bajas que el promedio. En cuarto, en todas las ciudades los datos se capturaron utilizando un programa único. Se hicieron modificaciones al programa solo cuando las peculiaridades de cada país lo hicieron necesario. En quinto lugar, una vez que los datos brutos se digitalizaron y organizaron utilizando un programa maestro, se procedió a la limpieza y estandarización de los datos provenientes de todos los países, utilizando un proceso de varias etapas, en las oficinas centrales de la OPS. Los objetivos de este proceso fueron lograr coherencia, Corregir errores y omisiones, generar esquemas de codificación comparables $\mathrm{y}$, por último, producir un documento con los códigos por aplicar en las bases de datos de cada país.

La depuración de los datos se llevó a cabo en dos etapas. En la primera, los errores evidentes de codificación que infringían controles simples de cohe- rencia se corrigieron mediante la revisión y reingreso de los códigos originales en los cuestionarios. Al principio este procedimiento meticuloso se empleó solo cuando los errores eran demasiado obvios. En la segunda etapa, se escribieron varios programas de coherencia. No se efectuó ninguna imputación durante el proceso de validación de los datos. Terminada la etapa de depuración de los datos se procedió a calcular los errores muestrales y los efectos de diseño. El producto final fue un conjunto de bases de datos estandarizados que son comparables y lle- 
CUADRO 6. Variables seleccionadas descriptivas de la población encuestada en Santiago (Chile), por sexo

\begin{tabular}{|c|c|c|c|c|c|c|}
\hline Variable & $\begin{array}{l}\text { Estimador } \\
\text { MUJER }\end{array}$ & $\begin{array}{c}\text { Error } \\
\text { estándar }\end{array}$ & IC95\% ${ }^{a}$ & $\begin{array}{l}\text { Estimador } \\
\text { HOMBRE }\end{array}$ & $\begin{array}{c}\text { Error } \\
\text { estándar }\end{array}$ & IC95\% \\
\hline \multicolumn{7}{|l|}{ Edad (años) } \\
\hline $60-64$ & 0,273 & 0,021 & $0,232-0,313$ & 0,329 & 0,031 & $0,268-0,390$ \\
\hline $65-74$ & 0,424 & 0,022 & $0,381-0,467$ & 0,434 & 0,032 & $0,372-0,496$ \\
\hline 75 o más & 0,303 & 0,019 & $0,266-0,341$ & 0,237 & 0,031 & $0,177-0,297$ \\
\hline \multicolumn{7}{|l|}{ Escolaridad } \\
\hline Sin educación formal & 0,115 & 0,013 & $0,091-0,139$ & 0,074 & 0,015 & $0,046-0,103$ \\
\hline Primaria & 0,212 & 0,018 & $0,177-0,247$ & 0,174 & 0,023 & $0,129-0,220$ \\
\hline Secundaria o más & 0,459 & 0,022 & $0,416-0,502$ & 0,505 & 0,033 & $0,441-0,570$ \\
\hline \multicolumn{7}{|l|}{ Estado civil } \\
\hline Nunca casado & 0,087 & 0,011 & $0,064-0,109$ & 0,039 & 0,012 & $0,016-0,063$ \\
\hline En unión libre & 0,419 & 0,023 & $0,374-0,463$ & 0,772 & 0,024 & $0,725-0,819$ \\
\hline Divorciado, separado, viudo & 0,482 & 0,022 & $0,440-0,525$ & 0,175 & 0,020 & $0,134-0,215$ \\
\hline \multicolumn{7}{|l|}{ Fuerza de trabajo } \\
\hline Activo & 0,138 & 0,015 & $0,109-0,167$ & 0,414 & 0,032 & $0,351-0,476$ \\
\hline \multicolumn{7}{|l|}{ Estado de salud declarado } \\
\hline Muy bueno & 0,036 & 0,008 & $0,021-0,052$ & 0,096 & 0,018 & $0,060-0,132$ \\
\hline Bueno & 0,303 & 0,021 & $0,263-0,344$ & 0,346 & 0,033 & $0,282-0,410$ \\
\hline Regular & 0,437 & 0,022 & $0,394-0,480$ & 0,392 & 0,031 & $0,330-0,453$ \\
\hline Malo & 0,223 & 0,018 & $0,189-0,257$ & 0,166 & 0,022 & $0,123-0,209$ \\
\hline \multicolumn{7}{|l|}{ Salud mental } \\
\hline Depresión & 0,295 & 0,020 & $0,256-0,334$ & 0,189 & 0,023 & $0,143-0,235$ \\
\hline Deterioro cognoscitivo & 0,131 & 0,014 & $0,103-0,158$ & 0,093 & 0,018 & $0,058-0,128$ \\
\hline \multicolumn{7}{|l|}{ Problemas de salud crónicos } \\
\hline Bajo peso & 0,013 & 0,005 & $0,004-0,021$ & 0,007 & 0,004 & $-0,001-0,015$ \\
\hline Obesidad & 0,320 & 0,021 & $0,280-0,360$ & 0,251 & 0,028 & $0,196-0,306$ \\
\hline Artritis & 0,401 & 0,022 & $0,359-0,444$ & 0,132 & 0,023 & $0,087-0,177$ \\
\hline Hipertensión & 0,556 & 0,022 & $0,513-0,599$ & 0,450 & 0,032 & $0,387-0,514$ \\
\hline Diabetes & 0,141 & 0,016 & $0,110-0,172$ & 0,120 & 0,021 & $0,079-0,162$ \\
\hline Cáncer & 0,057 & 0,010 & $0,037-0,077$ & 0,018 & 0,006 & $0,006-0,029$ \\
\hline Enfermedades pulmonares & 0,108 & 0,013 & $0,082-0,134$ & 0,136 & 0,020 & $0,096-0,176$ \\
\hline Enfermedades del corazón & 0,353 & 0,021 & $0,311-0,394$ & 0,278 & 0,027 & $0,224-0,332$ \\
\hline \multicolumn{7}{|l|}{ Discapacidad } \\
\hline AVDb (1 o más) & 0,251 & 0,018 & $0,216-0,286$ & 0,173 & 0,023 & $0,126-0,219$ \\
\hline AIVD (1 o más) & 0,286 & 0,019 & $0,249-0,323$ & 0,152 & 0,022 & $0,109-0,194$ \\
\hline \multicolumn{7}{|l|}{ Arreglos domiciliarios } \\
\hline Solo & 0,106 & 0,011 & $0,084-0,128$ & 0,063 & 0,011 & $0,040-0,085$ \\
\hline Con pareja & 0,413 & 0,023 & $0,368-0,457$ & 0,766 & 0,024 & $0,719-0,813$ \\
\hline Con otras personas & 0,481 & 0,022 & $0,438-0,524$ & 0,171 & 0,021 & $0,129-0,213$ \\
\hline
\end{tabular}

van al mínimo las idiosincrasias nacionales o locales.

\section{DESCRIPCIÓN DE LA POBLACIÓN ENCUESTADA}

En esta sección se ofrece un resumen de algunos de los resultados de la encuesta. No se intentó postular hipótesis ni brindar una perspectiva comparativa para examinar las diferencias y semejanzas entre ciudades. En varios artículos de este número especial de la Revista
Panamericana de Salud Pública se analizan diferentes aspectos de la salud y el bienestar de la población de 60 años y más, utilizando la base de datos SABE. La intención es, más bien, mostrar características importantes que sobresalen dentro de cada uno de los módulos más importantes del cuestionario, e investigar las características demográficas y sociales, arreglos domiciliarios, participación en la fuerza laboral y estado de salud física y mental.

Lo que sigue es una descripción de las variables demográficas y de salud de la población encuestada, en las siete ciudades incluidas en la muestra. En las figuras 1 y 2 , y en el cuadro 2, se destaca el contraste entre las distribuciones de edad jóvenes -en México, D.F. y São Paulo- y las distribuciones más viejas-en Bridgetown, Buenos Aires y Montevideo. Estos contrastes son una función de los antecedentes de disminución de la fecundidad y la mortalidad en la Región. Los países que experimentaron una transición tardía muestran más bien distribuciones jóvenes de su población mayor de 
CUADRO 7. Variables seleccionadas descriptivas de la población encuestada en La Habana (Cuba), por sexo

\begin{tabular}{|c|c|c|c|c|c|c|}
\hline Variable & $\begin{array}{l}\text { Estimador } \\
\text { MUJER }\end{array}$ & $\begin{array}{c}\text { Error } \\
\text { estándar }\end{array}$ & IC95\% ${ }^{a}$ & $\begin{array}{l}\text { Estimador } \\
\text { HOMBRE }\end{array}$ & $\begin{array}{c}\text { Error } \\
\text { estándar }\end{array}$ & IC95\% \\
\hline \multicolumn{7}{|l|}{ Edad (años) } \\
\hline 60-64 & 0,262 & 0,014 & $0,236-0,289$ & 0,304 & 0,019 & $0,266-0,342$ \\
\hline $65-74$ & 0,398 & 0,015 & $0,368-0,428$ & 0,431 & 0,020 & $0,392-0,471$ \\
\hline 75 o más & 0,340 & 0,014 & $0,313-0,367$ & 0,265 & 0,017 & $0,231-0,299$ \\
\hline \multicolumn{7}{|l|}{ Escolaridad } \\
\hline Sin educación formal & 0,051 & 0,006 & $0,039-0,063$ & 0,040 & 0,008 & $0,024-0,056$ \\
\hline Primaria & 0,560 & 0,017 & $0,526-0,595$ & 0,434 & 0,019 & $0,395-0,472$ \\
\hline Secundaria o más & 0,384 & 0,018 & $0,349-0,419$ & 0,526 & 0,020 & $0,488-0,565$ \\
\hline \multicolumn{7}{|l|}{ Estado civil } \\
\hline Nunca casado & 0,032 & 0,005 & $0,023-0,041$ & 0,029 & 0,007 & $0,016-0,042$ \\
\hline En unión libre & 0,230 & 0,013 & $0,205-0,255$ & 0,644 & 0,021 & $0,603-0,685$ \\
\hline Divorciado, separado, viudo & 0,737 & 0,013 & $0,711-0,762$ & 0,325 & 0,020 & $0,285-0,365$ \\
\hline \multicolumn{7}{|l|}{ Fuerza de trabajo } \\
\hline Activo & 0,079 & 0,008 & $0,063-0,095$ & 0,350 & 0,020 & $0,310-0,389$ \\
\hline \multicolumn{7}{|l|}{ Estado de salud declarado } \\
\hline Muy bueno & 0,044 & 0,007 & $0,030-0,057$ & 0,059 & 0,011 & $0,038-0,081$ \\
\hline Bueno & 0,240 & 0,013 & $0,214-0,266$ & 0,370 & 0,020 & $0,330-0,409$ \\
\hline Regular & 0,477 & 0,013 & $0,451-0,504$ & 0,429 & 0,021 & $0,387-0,471$ \\
\hline Malo & 0,138 & 0,010 & $0,117-0,158$ & 0,093 & 0,012 & $0,069-0,116$ \\
\hline \multicolumn{7}{|l|}{ Salud mental } \\
\hline Depresión & 0,236 & 0,013 & $0,209-0,262$ & 0,121 & 0,014 & $0,093-0,149$ \\
\hline Deterioro cognoscitivo & 0,127 & 0,011 & $0,105-0,149$ & 0,065 & 0,009 & $0,047-0,083$ \\
\hline \multicolumn{7}{|l|}{ Problemas de salud crónicos } \\
\hline Bajo peso & 0,070 & 0,008 & $0,053-0,086$ & 0,099 & 0,011 & $0,076-0,121$ \\
\hline Obesidad & 0,175 & 0,118 & $0,152-0,199$ & 0,056 & 0,009 & $0,038-0,074$ \\
\hline Artritis & 0,669 & 0,014 & $0,642-0,697$ & 0,396 & 0,020 & $0,356-0,436$ \\
\hline Hipertensión & 0,498 & 0,015 & $0,468-0,528$ & 0,358 & 0,018 & $0,322-0,394$ \\
\hline Diabetes & 0,200 & 0,013 & $0,175-0,225$ & 0,073 & 0,010 & $0,053-0,092$ \\
\hline Cáncer & 0,036 & 0,006 & $0,025-0,047$ & 0,030 & 0,006 & $0,019-0,042$ \\
\hline Enfermedades pulmonares & 0,128 & 0,011 & $0,106-0,149$ & 0,131 & 0,013 & $0,104-0,157$ \\
\hline Enfermedades del corazón & 0,274 & 0,014 & $0,247-0,302$ & 0,193 & 0,016 & $0,162-0,224$ \\
\hline \multicolumn{7}{|l|}{ Discapacidad } \\
\hline AVDb (1 o más) & 0,224 & 0,012 & $0,201-0,248$ & 0,141 & 0,013 & $0,115-0,168$ \\
\hline AIVD (1 o más) & 0,246 & 0,014 & $0,219-0,273$ & 0,144 & 0,015 & $0,115-0,173$ \\
\hline \multicolumn{7}{|l|}{ Arreglos domiciliarios } \\
\hline Solo & 0,119 & 0,010 & $0,098-0,139$ & 0,091 & 0,010 & $0,072-0,111$ \\
\hline Con pareja & 0,223 & 0,013 & $0,198-0,249$ & 0,624 & 0,020 & $0,583-0,666$ \\
\hline Con otras personas & 0,658 & 0,015 & $0,630-0,687$ & 0,284 & 0,020 & $0,245-0,324$ \\
\hline
\end{tabular}

60 años, mientras que sus predecesores muestran indicios de distribuciones mucho más viejas para este grupo de adultos mayores.

Cuando se segrega por edad y sexo, el estado civil de los adultos mayores corrobora la información censal en el sentido de que la proporción de adultos mayores sin pareja (separados, divorciados, viudos) se incrementa notablemente con la edad y que las tasas de incremento (por edad) y los niveles absolutos de esta variable son, con mucho, más grandes en las mujeres que en los varones. En el caso de La Habana, por ejemplo, casi $90 \%$ de las mujeres mayores de 75 años no tienen pareja, frente a $43 \%$ de los varones de los mismos grupos de edad. Estos contrastes se reproducen en otras ciudades que, en conjunto, muestran una gran similitud entre sí.

A pesar de que la participación en la fuerza laboral disminuye notablemente con la edad en todos los países, las tasas de adultos mayores que trabajan son bastante altas. Las tasas de participación en la fuerza laboral fluctúan entre 40 y $80 \%$ entre los varones del grupo de 60 a 64 años de edad, pero se reducen a la mitad de estas cifras entre las mujeres de las mismas edades. Mientras que en algunos países, como Uruguay, la edad en que disminuye la participación laboral es relativamente joven (menos de 65 años), en otros, como México, la salida del mercado laboral se produce más tarde y tiende a ser más gradual. Como se aprecia en los cuadros 3 a 9, la disparidad en la participación laboral entre los dos sexos es universal y ocurre en la dirección espe- 
CUADRO 8. Variables seleccionadas descriptivas de la población encuestada en la Ciudad de México (México), por sexo

\begin{tabular}{|c|c|c|c|c|c|c|}
\hline Variable & $\begin{array}{c}\text { Estimador } \\
\text { MUJER }\end{array}$ & $\begin{array}{c}\text { Error } \\
\text { estándar }\end{array}$ & IC95\% ${ }^{a}$ & $\begin{array}{l}\text { Estimador } \\
\text { HOMBRE }\end{array}$ & $\begin{array}{c}\text { Error } \\
\text { estándar }\end{array}$ & IC95\% \\
\hline \multicolumn{7}{|l|}{ Edad (años) } \\
\hline $60-64$ & 0,295 & 0,018 & $0,260-0,330$ & 0,361 & 0,023 & $0,316-0,406$ \\
\hline $65-74$ & 0,436 & 0,017 & $0,401-0,470$ & 0,436 & 0,022 & $0,391-0,480$ \\
\hline 75 o más & 0,270 & 0,016 & $0,238-0,302$ & 0,203 & 0,017 & $0,170-0,237$ \\
\hline \multicolumn{7}{|l|}{ Escolaridad } \\
\hline Sin educación formal & 0,213 & 0,020 & $0,174-0,252$ & 0,151 & 0,019 & $0,113-0,189$ \\
\hline Primaria & 0,578 & 0,021 & $0,537-0,619$ & 0,585 & 0,027 & $0,533-0,638$ \\
\hline Secundaria o más & 0,193 & 0,023 & $0,147-0,238$ & 0,250 & 0,028 & $0,196-0,305$ \\
\hline \multicolumn{7}{|l|}{ Estado civil } \\
\hline Nunca casado & 0,057 & 0,009 & $0,040-0,075$ & 0,019 & 0,006 & $0,006-0,031$ \\
\hline En unión libre & 0,387 & 0,017 & $0,353-0,422$ & 0,768 & 0,019 & $0,731-0,805$ \\
\hline Divorciado, separado, viudo & 0,552 & 0,018 & $0,515-0,588$ & 0,212 & 0,018 & $0,176-0,247$ \\
\hline \multicolumn{7}{|l|}{ Fuerza de trabajo } \\
\hline Activo & 0,154 & 0,014 & $0,127-0,181$ & 0,467 & 0,023 & $0,421-0,512$ \\
\hline \multicolumn{7}{|l|}{ Estado de salud declarado } \\
\hline Muy bueno & 0,054 & 0,009 & $0,036-0,072$ & 0,091 & 0,015 & $0,060-0,121$ \\
\hline Bueno & 0,225 & 0,017 & $0,190-0,259$ & 0,246 & 0,019 & $0,208-0,284$ \\
\hline Regular & 0,502 & 0,021 & $0,461-0,543$ & 0,497 & 0,024 & $0,450-0,543$ \\
\hline Malo & 0,216 & 0,016 & $0,186-0,247$ & 0,162 & 0,016 & $0,130-0,194$ \\
\hline \multicolumn{7}{|l|}{ Salud mental } \\
\hline Depresión & 0,213 & 0,015 & $0,183-0,243$ & 0,148 & 0,015 & $0,118-0,179$ \\
\hline Deterioro cognoscitivo & 0,220 & 0,017 & $0,186-0,253$ & 0,139 & 0,018 & $0,102-0,175$ \\
\hline \multicolumn{7}{|l|}{ Problemas de salud crónicos } \\
\hline Bajo peso & 0,007 & 0,003 & $0,001-0,013$ & 0,005 & 0,004 & $-0,002-0,013$ \\
\hline Obesidad & 0,304 & 0,020 & $0,264-0,343$ & 0,163 & 0,183 & $0,127-0,199$ \\
\hline Artritis & 0,311 & 0,019 & $0,273-0,350$ & 0,159 & 0,017 & $0,126-0,192$ \\
\hline Hipertensión & 0,488 & 0,019 & $0,450-0,527$ & 0,348 & 0,023 & $0,302-0,394$ \\
\hline Diabetes & 0,209 & 0,016 & $0,177-0,241$ & 0,224 & 0,020 & $0,184-0,263$ \\
\hline Cáncer & 0,025 & 0,006 & $0,013-0,037$ & 0,010 & 0,005 & $0,001-0,019$ \\
\hline Enfermedades pulmonares & 0,111 & 0,013 & $0,085-0,138$ & 0,081 & 0,012 & $0,058-0,104$ \\
\hline Enfermedades del corazón & 0,101 & 0,011 & $0,080-0,122$ & 0,098 & 0,014 & $0,070-0,127$ \\
\hline \multicolumn{7}{|l|}{ Discapacidad } \\
\hline AVDb (1 o más) & 0,214 & 0,016 & $0,182-0,246$ & 0,161 & 0,018 & $0,126-0,197$ \\
\hline AIVDc (1 o más) & 0,286 & 0,017 & $0,252-0,320$ & 0,144 & 0,016 & $0,112-0,177$ \\
\hline \multicolumn{7}{|l|}{ Arreglos domiciliarios } \\
\hline Solo & 0,117 & 0,013 & $0,092-0,142$ & 0,073 & 0,011 & $0,051-0,096$ \\
\hline Con pareja & 0,379 & 0,018 & $0,344-0,414$ & 0,750 & 0,020 & $0,710-0,790$ \\
\hline Con otras personas & 0,505 & 0,020 & $0,465-0,544$ & 0,177 & 0,018 & $0,141-0,213$ \\
\hline
\end{tabular}

Fuente: Organización Panamericana de la Salud. Encuesta SABE, 2001 e informes preliminares.

a IC95\%: intervalo de confianza de 95\%.

${ }^{\text {b }}$ Actividades de la vida diaria.

${ }^{c}$ Actividades instrumentales de la vida diaria.

rada según el conocimiento que se tiene de la participación en el mercado de trabajo de este grupo de población.

Es muy probable que los adultos mayores permanezcan en la fuerza de trabajo $-\mathrm{y}$ aun que reingresen después de retirarse-, por necesidad más que por elección. Parte de este aspecto se aborda mediante el examen de la relación que existe entre la participación en la fuerza laboral y los ingresos por pensiones. En particular en casi todas las ciudades, con la excepción de México, D.F. y São Paulo, la fracción de varones que reciben algún tipo de pensión es muy cercana a $80 \%$. Además, las disparidades por sexo en los ingresos por pensiones son acentuadas y universales. Como es de esperar, solo una minoría de las mujeres tiene ingresos por pensiones.

Dada la disparidad en la mortalidad entre varones y mujeres, en todas las edades las mujeres están siempre más propensas a vivir sin pareja en la vejez que los varones. Resulta de interés el bajo porcentaje de mujeres que viven solas en las ciudades estudiadas. $\mathrm{Al}$ se- gregar por grupos de edad, se advierte que los patrones son constantes, en vez de aumentar o fluctuar con la edad, como se esperaría si la propensión a vivir solo respondiera en parte a las diferencias en la edad de los hijos. En realidad, mientras que en Buenos Aires y Montevideo la proporción de mujeres que viven solas excede de $25 \%$, en La Habana y São Paulo la cifra está por debajo de 15\%; las otras ciudades ocupan lugares intermedios. A partir de estos patrones observados, no está claro si las diferencias entre 
CUADRO 9. Variables seleccionadas descriptivas de la población encuestada en Montevideo (Uruguay), por sexo

\begin{tabular}{|c|c|c|c|c|c|c|}
\hline Variable & $\begin{array}{l}\text { Estimador } \\
\text { MUJER }\end{array}$ & $\begin{array}{c}\text { Error } \\
\text { estándar }\end{array}$ & IC95\% ${ }^{a}$ & $\begin{array}{l}\text { Estimador } \\
\text { HOMBRE }\end{array}$ & $\begin{array}{c}\text { Error } \\
\text { estándar }\end{array}$ & IC95\% \\
\hline \multicolumn{7}{|l|}{ Edad (años) } \\
\hline 60-64 & 0,219 & 0,017 & $0,186-0,252$ & 0,200 & 0,018 & $0,164-0,237$ \\
\hline $65-74$ & 0,471 & 0,018 & $0,435-0,507$ & 0,515 & 0,023 & $0,470-0,560$ \\
\hline 75 o más & 0,310 & 0,017 & $0,278-0,343$ & 0,285 & 0,024 & $0,237-0,333$ \\
\hline \multicolumn{7}{|l|}{ Escolaridad } \\
\hline Sin educación formal & 0,050 & 0,008 & $0,036-0,065$ & 0,026 & 0,009 & $0,009-0,043$ \\
\hline Primaria & 0,672 & 0,020 & $0,633-0,711$ & 0,645 & 0,027 & $0,593-0,698$ \\
\hline Secundaria o más & 0,274 & 0,020 & $0,234-0,313$ & 0,327 & 0,025 & $0,277-0,377$ \\
\hline \multicolumn{7}{|l|}{ Estado civil } \\
\hline Nunca casado & 0,036 & 0,007 & $0,023-0,049$ & 0,033 & 0,008 & $0,017-0,049$ \\
\hline En unión libre & 0,352 & 0,020 & $0,312-0,393$ & 0,720 & 0,026 & $0,667-0,772$ \\
\hline Divorciado, separado, viudo & 0,610 & 0,021 & $0,568-0,652$ & 0,239 & 0,023 & $0,192-0,285$ \\
\hline \multicolumn{7}{|l|}{ Fuerza de trabajo } \\
\hline Activo & 0,115 & 0,012 & $0,092-0,139$ & 0,204 & 0,023 & $0,158-0,250$ \\
\hline \multicolumn{7}{|l|}{ Estado de salud declarado } \\
\hline Muy bueno & 0,160 & 0,014 & $0,133-0,188$ & 0,189 & 0,018 & $0,154-0,225$ \\
\hline Bueno & 0,406 & 0,017 & $0,373-0,439$ & 0,501 & 0,026 & $0,450-0,552$ \\
\hline Regular & 0,349 & 0,015 & $0,318-0,379$ & 0,268 & 0,024 & $0,220-0,316$ \\
\hline Malo & 0,085 & 0,012 & $0,061-0,109$ & 0,042 & 0,009 & $0,023-0,060$ \\
\hline \multicolumn{7}{|l|}{ Salud mental } \\
\hline Depresión & 0,222 & 0,016 & $0,191-0,254$ & 0,113 & 0,018 & $0,077-0,148$ \\
\hline Deterioro cognoscitivo & 0,021 & 0,005 & $0,012-0,030$ & 0,014 & 0,006 & $0,003-0,025$ \\
\hline \multicolumn{7}{|l|}{ Problemas de salud crónicos } \\
\hline Bajo peso & 0,160 & 0,014 & $0,133-0,188$ & 0,037 & 0,009 & $0,020-0,054$ \\
\hline Obesidad & 0,406 & 0,017 & $0,373-0,439$ & 0,174 & 0,024 & $0,127-0,221$ \\
\hline Artritis & 0,349 & 0,015 & $0,318-0,379$ & 0,321 & 0,024 & $0,272-0,370$ \\
\hline Hipertensión & 0,085 & 0,012 & $0,061-0,109$ & 0,377 & 0,022 & $0,332-0,421$ \\
\hline Diabetes & 0,160 & 0,014 & $0,133-0,188$ & 0,124 & 0,017 & $0,090-0,158$ \\
\hline Cáncer & 0,406 & 0,017 & $0,373-0,439$ & 0,045 & 0,009 & $0,027-0,062$ \\
\hline Enfermedades pulmonares & 0,349 & 0,015 & $0,318-0,379$ & 0,109 & 0,016 & $0,078-0,140$ \\
\hline Enfermedades del corazón & 0,085 & 0,012 & $0,061-0,109$ & 0,226 & 0,016 & $0,195-0,258$ \\
\hline \multicolumn{7}{|l|}{ Discapacidad } \\
\hline AVDb (1 o más) & 0,210 & 0,014 & $0,181-0,238$ & 0,108 & 0,013 & $0,083-0,134$ \\
\hline AIVD (1 o más) & 0,163 & 0,013 & $0,137-0,190$ & 0,079 & 0,012 & $0,054-0,103$ \\
\hline \multicolumn{7}{|l|}{ Arreglos domiciliarios } \\
\hline Solo & 0,223 & 0,020 & $0,182-0,264$ & 0,128 & 0,018 & $0,093-0,163$ \\
\hline Con pareja & 0,316 & 0,021 & $0,273-0,358$ & 0,337 & 0,027 & $0,284-0,391$ \\
\hline Con otras personas & 0,461 & 0,020 & $0,422-0,500$ & 0,535 & 0,028 & $0,479-0,590$ \\
\hline
\end{tabular}

ciudades reflejan la variación en la disponibilidad de familiares, más que preferencias sociales o la disponibilidad de vivienda. Argentina y Uruguay, y hasta cierto punto Barbados, experimentaron transiciones de fecundidad y mortalidad más tempranas y, por tanto, es posible que en estos países los adultos mayores hayan tenido menos disponibilidad de familiares.

En cuanto al papel de la familia, cabe preguntar: ¿Hay signos de que tiendan a desaparecer los arreglos tradicionales en que los adultos mayores viven con uno o más de sus hijos? No hay punto de comparación para responder en definitiva esta pregunta y no es válido suponer que México, D.F. o La Habana tomarán el mismo rumbo que Buenos Aires y Montevideo. En cualquier caso, la proporción de adultos mayores que viven solos es menor en estos siete países que en los de Norteamérica y Europa Occidental, aunque más alta que en Asia (18).

Se sabe que el estado de salud y la participación en la fuerza laboral dependen, en una medida no trivial, del nivel educativo de los adultos mayores. Por esta razón, aquí se presentan cifras que muestran las distribuciones de los adultos mayores por escolaridad en todas las ciudades estudiadas. Se han elegido tres categorías: sin educación formal, primaria, y secundaria y más.

En todos los países los varones tienen una escolaridad más alta que las mujeres, y las cohortes viejas están generalmente en peores condiciones que las jóvenes. El patrón de desigualdades educacionales por edad se explica por las mejoras que se produjeron en el sistema educativo de la Región a principios de la segunda guerra mundial. Pero la característica más impor- 
FIGURA 1. Distribución porcentual de las mujeres adultas mayores, por grupos de edad y ciudades de estudio, 2001

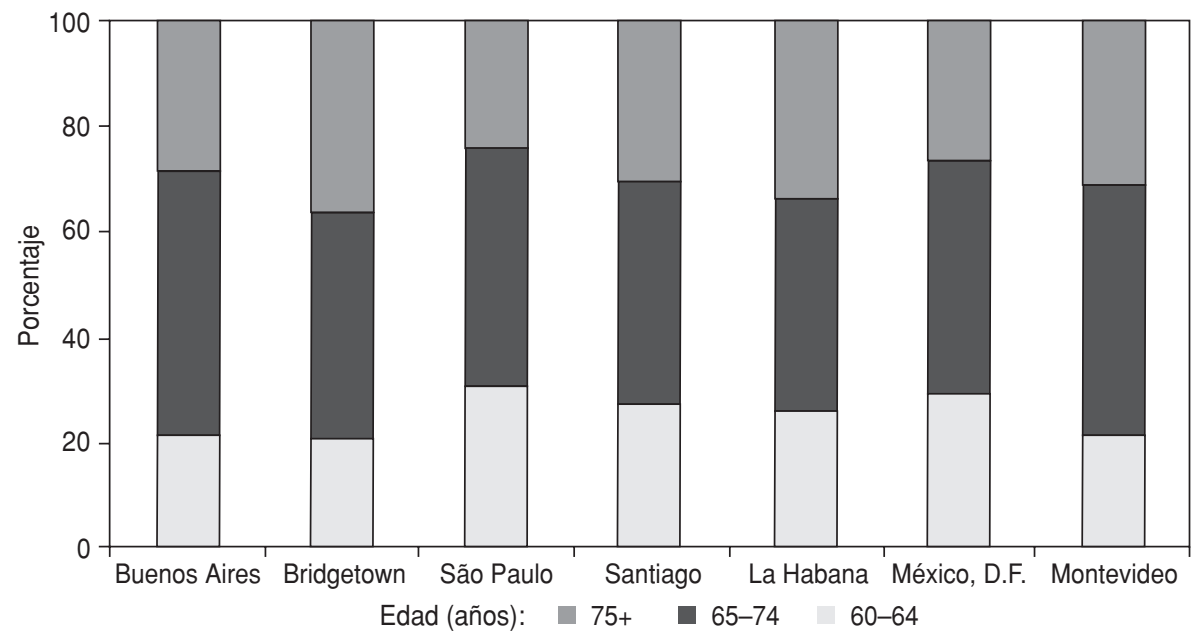

FIGURA 2. Distribución porcentual de los varones adultas mayores, por grupos de edad y ciudades de estudio, 2001

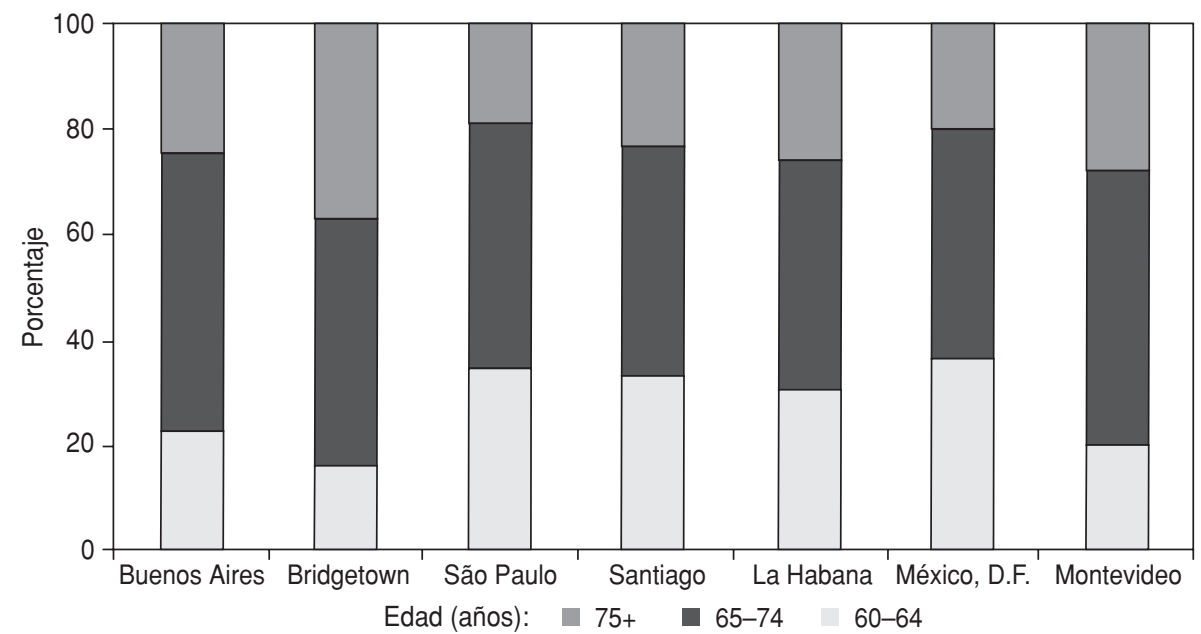

tante es el gran contraste que se advierte entre los países, en todas las edades y en ambos sexos. En La Habana, independientemente de la edad y el sexo, más de $50 \%$ de los adultos mayores caen en el grupo de escolaridad más alta, mientras que en São Paulo la cifra es menor de $20 \%$. Tales disparidades reflejan la existencia de instituciones y políticas distintas que en algunos casos probablemente facili- tencia de sistemas educativos que promueven con mayor eficiencia una educación superior puede acompañarse del desarrollo de medios públicos que actúen a favor de los adultos mayores en áreas no relacionadas con los logros educacionales individuales. Una tarea pendiente es la identificación apropiada de los efectos que dependen de las características individuales y los que pertenecen al contexto social.

En cuanto a problemas crónicos de salud, la encuesta SABE ha puesto de relieve la aparición de determinadas enfermedades, como hipertensión, trastornos cardiovasculares, artritis y diabetes que, en este orden, prevalecen entre los adultos mayores. Se observan importantes diferencias por sexo: las mujeres sufren más artritis que los varones y existe un notable contraste entre países; por ejemplo, en México, D.F. y en Bridgetown se observan prevalencias más altas de diabetes que en otras ciudades. En otros estudios se ha demostrado que la prevalencia de los problemas de salud que en el estudio SABE se declaran - trastornos cardiovasculares, artritis y diabetes- es más alta que la señalada en adultos mayores en Norteamérica (3). Puesto que los trastornos crónicos notificados son aquellos que diagnosticó un médico o una enfermera, y que la población de adultos mayores de la Región afronta más problemas de acceso y calidad en la atención de la salud que sus semejantes en otros lugares, las diferencias observadas resultan alarmantes y, según se estima, la prevalencia real podría ser aun mayor que la que revela la encuesta SABE.

Debe destacarse también la necesidad de que al realizar comparaciones entre países se tengan en cuenta los contextos institucionales, las políticas sectoriales de salud y el desarrollo económico y educativo de los países en general y de las personas de 60 años y más en particular. Entender el contexto en el que las personas viven y funcionan es indispensable para hacer estudios comparativos entre países y contribuir a una mejor comprensión del envejecimiento en América Latina y el Caribe. 


\section{CONCLUSIONES}

La encuesta SABE constituye un punto de partida en el estudio sistemático del envejecimiento en zonas urbanas de la Región de América Latina y el Caribe, y ha sido instrumental en la articulación de una agenda de investigación para la Región (19). La encuesta SABE da fundamento a las recomendaciones siguientes: 1) el desarrollo futuro de la investigación sobre la salud y el envejecimiento debe guiarse por diseños multidisciplinarios. Es indispensable estudiar la salud de las personas adultas mayores en relación con su situación económica y familiar. La interacción entre epidemiólogos, economistas y sociólogos en el diseño de encuestas de envejecimiento es indispensable; 2) para lograr bases de datos comparables entre países es necesario llegar a un consenso en la Región sobre los instrumentos de evaluación apropiados para cada aspecto de la salud y el bienestar en las personas adultas mayores, y utilizarlas de manera uniforme en futuras investigaciones; 3 ) los estudios que per- miten comparaciones entre ciudades y entre países que tienen contextos socioeconómicos, demográficos y políticos diferentes son importantes para obtener conocimientos más completos y sólidos; en cuyo caso las varianzas socioeconómicas permiten hacer inferencias más robustas que cuando se investiga en un solo contexto; 4) la investigación sobre la salud pública y el envejecimiento no justificará la inversión que requiere si no se establece un diálogo entre los investigadores y los políticos y tomadores de decisiones, de tal forma que las políticas de salud se guíen efectivamente por la evidencia.

Asimismo, el estudio multicéntrico SABE es solamente un primer paso. Para establecer tendencias y evaluar las relaciones complejas que prevalecen entre el envejecimiento y las condiciones en que ocurre, es necesario invertir en estudios longitudinales, interdisciplinarios y regionales (20). La complejidad de la organización de este tipo de encuesta supone un compromiso regional para la inversión y la coordinación de estos esfuerzos.
La Organización Panamericana de la Salud, con el apoyo del Centro Colaborador OMS/OPS del National Institute on Aging (Instituto Nacional de Envejecimiento, NIA) de Estados Unidos, ha contribuido en grado importante a promover una agenda de investigación sobre el tema de salud, bienestar y envejecimiento. Este número especial de la Revista Panamericana de Salud Pública es un ejemplo de los trabajos que se han generado con la base de datos de SABE. La comunidad científica tiene una base de datos importante para formular nuevas hipótesis, diseñar nuevos estudios y trabajar en nuevas redes, con el fin de obtener los conocimientos necesarios para promover una mayor longevidad satisfactoria en América Latina y el Caribe en el siglo XXI.

Agradecimiento. Los autores agradecen a Nuria Rodríguez, de la Universidad de Barcelona, España, su valioso auxilio en la elaboración de este documento.

\section{REFERENCIAS}

1. Preston $\mathrm{SH}$, Martin LG. Introduction. En: Martin LG, Preston SH, eds. Demography of aging. Washington D.C.: National Academy Press; 1994. Pp. 1-7.

2. Kinsella K, Martin LG. Research on the demography of aging in developing countries. En: Martin LG, Preston SH, eds. Demography of aging. Washington, D.C.: National Academy Press; 1994. Pp. 356-403.

3. Palloni A, Pinto G, Peláez, M. Demographic and health conditions of ageing in Latin America and the Caribbean. Int J Epidemiol. 2002;31:762-71.

4. Olshansky SJ, Carnes BA, Cassel CK. The aging of the human species. Sci Am. 1993; April:46-52.

5. Fries JF. The compression of morbidity. Milbank Mem Fund Q. 1983;61(3):397-419.

6. Singer BS, Manton K. What's the fuss about the compression morbidity? Chance. 1994; 7(4):21-30.

7. Crimmins E, Saito $Y$, Ingegneri D. Changes in life expectancy and disability-free life expectancy in the United States. Popul Dev Rev. 1989;15(2):235-68.

8. Manton, K. The dynamics of population aging: demography and policy analysis. Milbank Mem Q. 1991;69(2):309-38.
9. Wise, DA. Introduction. En: Wise DA, ed. Advances in the economics of aging. Chicago: University of Chicago Press; 1996. Pp. 1-10.

10. Lee R. The formal demography of population aging, transfers, and the economic life cycle. En: Martin LG, Preston SH, eds. Demography of aging. Washington, D.C.: National Academy Press; 1994. Pp. 8-49.

11. Manton K, Stallard E. A medical demography: interaction of disability and mortality. En: Martin LG, Preston SH, eds. Demography of aging. Washington, D. C.: National Academy Press; 1994. Pp. 217-78.

12. Lee R, Tuljapurkar S. Death and taxes: longer life, consumption and social security. Demography. 1997;34(1):67-81.

13. United States Senate. Aging America. Washington, D.C.: U.S. Senate Committee on Aging; 1991.

14. Mesle F, et al. Tendances récentes de la mortalité par cause en Russie 1965-1994. Donnés Statistiques, Institut National d'Études Démographiques. París: Institut National d'Études Démographiques; 1996.

15. Bobadilla JL, Costello C, Mitchell F. Premature death in the new independent states. Washington, D. C.: National Academy Press; 1997.
16. United Nations. World population ageing 1950-2050. New York: UN; 2002.

17. Barker DJP. Mothers, babies and health in later life. London: Churchill-Livingston; 1997.

18. Palloni A, Devos S, Peláez M. Aging in Latin America and the Caribbean. Washington, D.C.: PAHO Working Papers; 2000.

19. Peláez M, Palloni A. La salud en el envejecimiento: agenda de investigación para América Latina y el Caribe. En: Engler TA, Peláez M, eds. Más vale por viejo. Washington, D.C.: Banco Interamericano de Desarrollo; 2002.

20. National Research Council. Preparing for an aging world: the case for cross-national research. Washington, D.C: National Academy Press. 2001.

Manuscrito recibido el 28 de febrero de 2005. Aceptado para publicación, tras revisión, el 29 de marzo de 2005. 


\section{Red de Investigación sobre Envejecimiento en América Latina y el Caribe (REALCE)}

Durante los últimos cinco años se han hecho en América Latina y el Caribe tres importantes encuestas domiciliarias de propósitos múltiples y carácter poblacional, todas ellas enfocadas en adultos de edad avanzada. Son las siguientes:

1. Salud, Bienestar y Envejecimiento (SABE), encuesta multicéntrica de personas de 60 años de edad o mayores en Buenos Aires, Argentina, Bridgetown, Barbados, México, D.F., México, La Habana, Cuba, Montevideo, Uruguay, Santiago, Chile y São Paulo, Brasil. Se inició en octubre de 1999 y se terminó en junio de 2000 bajo el patrocinio de la Organización Panamericana de la Salud y con la colaboración de investigadores en universidades y ministerios de salud en cada ciudad e instituciones como el Banco Interamericano de Desarrollo, el Instituto Nacional de Envejecimiento de los Estados Unidos, el Fondo de Apoyo a la Investigación en São Paulo (FAPESP) y el Banco Caribeño de Desarrollo.

2. Estudio Nacional de Salud y Envejecimiento en México (ENASEM), en el que se llevó a cabo una encuesta representativa de la población nacional de personas de 50 años o mayores, en zonas urbanas y rurales. Las primeras entrevistas se llevaron a cabo en 2000 y las mismas personas fueron reentrevistadas en 2003.

3. Condiciones de Salud de los Adultos de Edad Mayor en Puerto Rico (Puerto Rican Elderly: Health Conditions, PREHCO), encuesta representativa de personas de 60 años o mayores en zonas urbanas y rurales. La primera entrevista, que abarcó a 4293 puertorriqueños de 60 años o más y sus cónyuges, se completó en 2003 y las mismas personas se reentrevistarán en 2006.

Los últimos dos estudios -ENASEM y PREHCO - fueron financiados por el Instituto Nacional de Envejecimiento (National Institute on Aging, NIA), que forma parte de los Institutos Nacionales de Salud (National Institutes of Health, NIH) en Bethesda, Maryland, Estados Unidos de América.

Todos los estudios mencionados — SABE, ENASEM y PREHCO— se diseñaron con el fin de poder hacer comparaciones entre uno y otro y con estudios realizados anteriormente en países desarrollados utilizando indicadores y parámetros de medición similares. Todas las encuestan mantienen un conjunto de preguntas comparables y los mismos consultores expertos han participado en las tres. En todos los casos se ha promovido el acceso público a las bases de datos. Por su naturaleza, el estudio SABE permite hacer comparaciones entre las distintas ciudades abarcadas en él, mientras que el estudio ENASEM en México y el PREHCO en Puerto Rico permiten hacer comparaciones entre la población que reside dentro de cada país y la que es originaria de dichos países pero reside actualmente en Estados Unidos. 
Estos estudios, más los que ya se han realizado o están en marcha en Asia, Estados Unidos y Europa, ofrecen la oportunidad de aprender mucho acerca de la situación en que envejecen las personas en diferentes partes del mundo. Se considera que estos estudios pueden ser informativos e integradores, aunque sus resultados aún no han sido sometidos a suficiente análisis como para poder hacer comparaciones transnacionales.

Estos notables esfuerzos por recolectar datos de una primera generación requerirán análisis desde diversos puntos de vista. Los investigadores que han tomado parte en estos y otros estudios reconocen que la labor de investigación en la Región apenas se ha iniciado. Por ejemplo, la mayoría de los países aún no cuentan con estudios de carácter nacional a nivel poblacional que permitan documentar las medidaas necesarias para maximizar el bienestar de los adultos mayores. Asimismo, hay que continuar los esfuerzos en algunos países por incluir en los estudios a las areas rurales, así como a grupos minoritarios, entre ellos la población indígena. También se reconoce la necesidad de seguir fomentando los estudios longitudinales.

En los últimos cinco años los investigadores interesados en la situación de salud de los adultos mayores se han reunido en diversos foros científicos para uniformar criterios, compartir lecciones, y asesorarse mutuamente acerca de los diferentes proyectos. Durante sus diversas discusiones se ha detectado la necesidad de integrar formalmente un vehículo para difundir las actividades de investigación, compartir datos y resultados, y fomentar la colaboración entre investigadores en distintos países con interés en el envejecimiento de la población de la Región. Con esa finalidad se decidió formar una red de investigación encaminada a facilitar el acceso a información y a hacer más eficiente el trabajo de investigación. De ahí surgió en 2004-2005 la Red de Investigación sobre Envejecimiento en América Latina y el Caribe (REALCE), integrada por un grupo de investigadores deseosos de estudiar los aspectos sociales, económicos, sanitarios, y de bienestar y calidad de vida del proceso de envejecimiento de la población de la Región, incluida la de origen latinoamericano y caribeño con residencia en Estados Unidos y Canadá.

La Red, que desde sus comienzos ha tenido el apoyo de la Organización Panamericana de la Salud y del NIA, busca maximizar la capacidad de investigación en la Región mediante la difusión de información acerca de investigaciones o sus resultados, la disponibilidad de datos y los sitios donde pueden consultarse, conferencias y otros eventos profesionales próximos a celebrarse, y oportunidades de capacitación o perfeccionamiento profesional. Además, la Red busca fomentar proyectos de colaboración entre investigadores e impulsar la organización de conferencias regionales sobre temas de interés. La Red tiene su sitio de Internet en la dirección www.REALCE.org

Se espera que muchos más investigadores se unan a los esfuerzos realizados hasta ahora en la Región, a fin de seguir apoyando la generación de datos y conocimientos, así como la formación de recursos humanos. Todo ello tiene por objeto preparar y modificar la infraestructura social, económica y política para hacerles frente de la mejor manera posible a las nuevas necesidades que plantea el envejecimiento de la población mundial. 
ABSTRACT This document outlines the methodology of the Salud, Bienestar y Envejecimiento (Health, Well-Being, and Aging) survey (known as the "SABE survey"), and it also summarizes the challenges that the rapid aging of the population in Latin America and the

The Health, Well-Being, Caribbean imposes on society in general and especially on health services. The popuand Aging ("SABE") survey: methodology applied and profile of the study population lations of the countries of Latin America and the Caribbean are aging at a rate that has not been seen in the developed world. The evaluation of health problems and disability among older adults in those countries indicates that those persons are aging with more functional limitations and worse health than is true for their counterparts in developed nations. In addition, family networks in Latin America and the Caribbean are changing rapidly and have less capacity to make up for the lack of protections provided by social institutions. The multicenter SABE study was developed with the objective of evaluating the state of health of older adults in seven cities of Latin America and the Caribbean: Bridgetown, Barbados; Buenos Aires, Argentina; Havana, Cuba; Mexico City, Mexico; Montevideo, Uruguay; Santiago, Chile; and São Paulo, Brazil. The SABE survey has established the starting point for systematic research on aging in urban areas of Latin America and the Caribbean. Comparative studies of these characteristics and with this comparative nature should be extended to other countries, areas, and regions of the world in order to expand the knowledge available on older adults.

Keywords Health, quality of life, aging, population, Latin America, Caribbean Region. 